\title{
Additivity and Interaction Between Size Ratio and Response Category in the Comparison of Size-Discrepant Shapes
}

\author{
Pierre Jolicoeur and Derek Besner \\ University of Waterloo, Waterloo, Ontario, Canada
}

\begin{abstract}
In Experiment 1, subjects made same-different judgments to pairs of shapes that could differ (irrelevantly) in size and in which different pairs combined distinct shapes. Size discrepancy had an effect both on same and different responses. However, the effect on different responses was not monotonic across size discrepancies. It is argued that this nonmonotonicity was produced by a form of bias acting to slow different responses for same-sized pairs. Consistent with the proposed bias account, the nonmonotonic size-discrepancy effect on different trials was eliminated in Experiment 2, in which trials were blocked by size ratio. In Experiment 3, subjects performed a task similar to that in Experiments 1 and 2. However, additional visual information was added inside the bounding contour of the shapes, and this information was either the same or different across shapes. The match between within-contour information across shapes (whether same or different) was varied orthogonally with whether the bounding contours of the shapes were the same or different. In this experiment subjects decided whether the bounding contours of the shapes were the same or different, while ignoring the added information within the contours. When the added information matched across the two shapes, same responses were facilitated relative to when the added information mismatched. The converse occurred for different responses. This effect was more pronounced when the shapes were shown at the same size than when the shapes were at different sizes. In general, the results suggest that (a) size discrepancy affects some perceptual operations that are preliminary to shape matching and (b) bias mechanisms can play an important role in shape-matching experiments in which the shapes can be shown in different sizes. The interaction of two processes-size scaling and bias-can account for these and hitherto contradictory results in the literature.
\end{abstract}

It is relatively easy for observers to consider two shapes that differ only in size as members of the same basic shape category. In fact, when shown the "same" shape at different sizes, often we immediately "see" that the shapes are identical except for the size difference. How is this perception of shape equivalence achieved? In this article we focus on how people decide that two shapes are the same or different while considering size as an irrelevant dimension.

In one model used to account for shape constancy across different sizes, it has been assumed that the visual system performs a continuous analog size scaling normalization on one shape to equate the sizes of the two shapes and that this transformed representation is then compared with the other shape (Bundesen \& Larsen, 1975; Corcoran \& Besner, 1975; Larsen, 1985; Larsen \& Bundesen, 1978; Posner \& Mitchell, 1967; Sekuler \& Nash, 1972). This approach has been motivated by the frequent finding that increasingly more time is required to decide that two shapes are the same as the ratio of the sizes of

Order of authorship was determined by relative exactitude of Bayesian priors. This research was supported by National Science and Engineering Research Council (NSERC) Grant A2579 awarded to Pierre Jolicoeur and by NSERC Grant A0998 awarded to Derek Besner.

We thank Keith McGowan, Dave Rynard, and Doug Snow for technical assistance.

Correspondence concerning this article should be addressed to Pierre Jolicoeur, Department of Psychology, University of Waterloo, Waterloo, Ontario, Canada N2L 3G1. the shapes is increased (Bundesen \& Larsen, 1975; Bundesen, Larsen, \& Farrell, 1981; Howard \& Kerst, 1978; Larsen, 1985; Larsen \& Bundesen, 1978; Sekuler \& Nash, 1972).

One difficulty with the size scaling model described above is that response times for different trials have sometimes been found not to increase monotonically as the size ratio is increased (Besner, 1983; Besner \& Coltheart, 1975, 1976). Because of this finding, Besner $(1978,1983$; Besner \& Coltheart, $1975,1976)$ argued that a preprocessing normalization explanation of the effect of size ratio on response time is inadequate, given that this approach leads to the prediction that the effect of size ratio should be identical for responses to same pairs and to different pairs. If the visual system must adjust the size of shapes so they are the same before they are compared, then the magnitude of the size-ratio effect should be equivalent for same and different responses. This prediction follows from the fact that the decision as to whether the shapes are the same or different occurs after the size scaling process has run to completion, and because the decision is presumed to be independent of the direction or magnitude of the scaling operation. Thus, the preprocessing explanation for the size-ratio effect predicts additive effects of size and response category, an outcome that is contradicted by the interaction sometimes found between size ratio and response category (Besner, 1983; Besner \& Coltheart, 1975, 1976).

Because the results for different trials can have important implications for models of the size-ratio effect, a closer examination of this issue seems warranted. As noted by Besner and Col- 

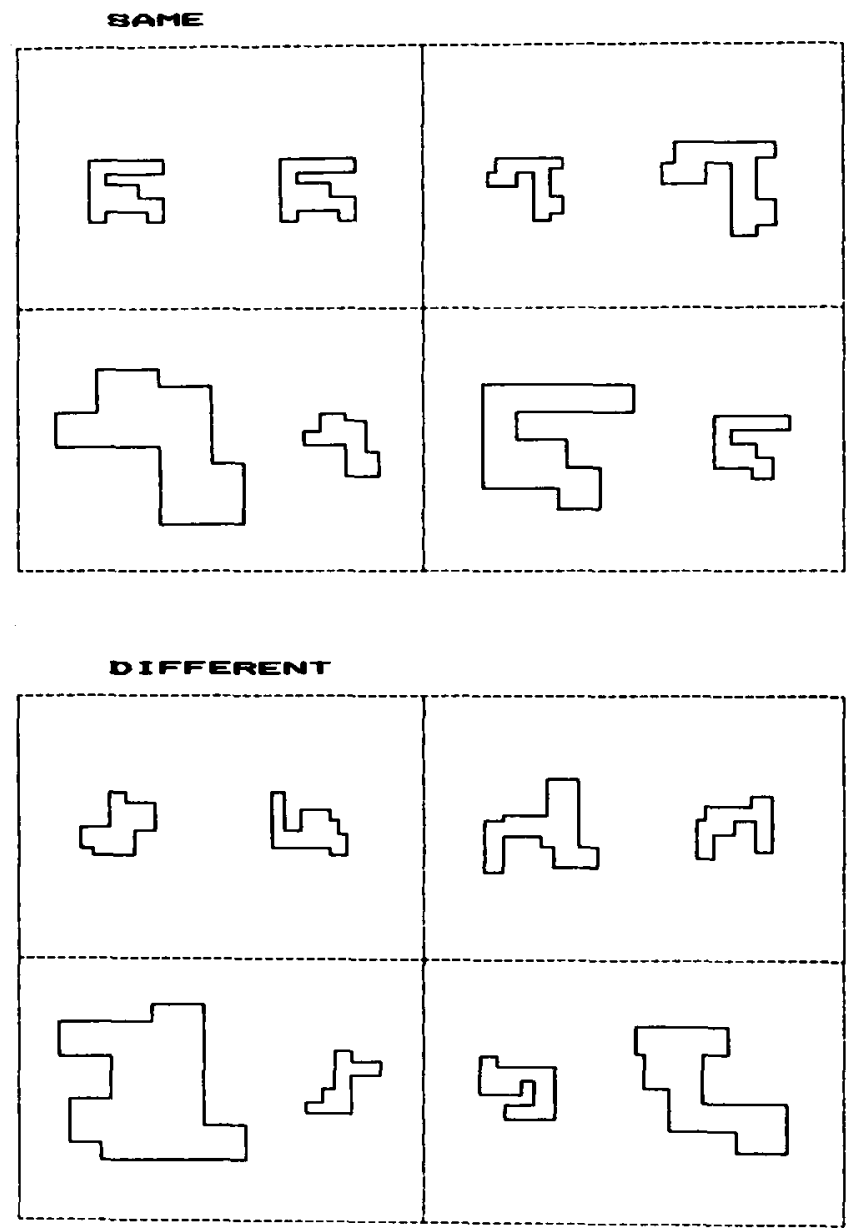

Figure 1. Example stimuli used in Experiments 1 and 2. (Same pairs are displayed in the top panel; different pairs are displayed in the bottom panel. In each panel one pair is shown using one of the four size ratios used in every experiment: The top-left quadrant of each panel contains a pair at size ratio $1: 1$; the top-right quadrant contains a pair at size ratio 1:1.5; the bottom-right quadrant contains a pair at size ratio $1: 2$; and the bottom-left quadrant contains a pair at size ratio 1:2.5. Note that the shapes were shown to subjects as white lines on a black background.)

theart (1976; see also Besner, 1983), experiments in which different trials consisted of a shape and a rotated version of the same shape (usually rotated by $180^{\circ}$ ) have yielded comparable effects of size ratio across same and different responses (e.g., Bundesen \& Larsen, 1975; Howard \& Kerst, 1978; Larsen, 1985; Larsen \& Bundesen, 1978). In contrast, the experiments that have revealed negligible effects of size ratio in different trials have used different shapes as members of the different pairs rather than the same shape rotated in the image plane (e.g., Besner, 1983; Besner \& Coltheart, 1975, 1976). (Note that Bundesen \& Larsen, 1975, Larsen, 1985, and Sekuler \& Nash, 1972, report experiments in which the different trials used pairs composed of different shapes; however, they do not report any analyses for these trials). Thus, it seems plausible that the composition of the different pairs could determine whether one observes a size-ratio effect on different responses (see Besner, 1983, for additional evidence). Unfortunately, a closer examination of the studies in which systematic increases in response times for different trials were not found reveals that they used relatively small size differences. Besner and Coltheart (1975) used size ratios of 1:1.5 and smaller. Besner and Coltheart (1976) used ratios between 1:1 and 1:1.67. Besner (1983; Experiment 3) used ratios of $1: 1$ and 1:1.5. Although in all of the above experiments there was a significant effect of size ratio on same responses, there was a minimal or inconsistent effect of size ratio for different responses. It seems possible that the inconsistent size-ratio effects reported for different pairs composed of different shapes (as opposed to rotated shapes) may be due, in part, to the small range of size ratios used in the experiments.

\section{Experiment 1}

The purpose of Experiment 1 was to determine whether the pattern of mean response times across size ratios would be additive across same and different responses when different pairs are formed by combining different shapes. We included size ratios ranging from $1: 1$ to $1: 2.5$, which is a larger range than in most previous studies that have reported results for different trials composed of different shapes.

\section{Method}

Subjects. The subjects were 20 undergraduates at the University of Waterloo who were paid for their participation. All subjects had normal or corrected-to-normal vision. No subject in this experiment participated in Experiment 2 or 3.

Stimuli. The stimuli were shapes made up of vertical and horizontal line segments connected from end to end so as to create a closed form. Twenty different shapes were created. Example stimuli are displayed in Figure 1. These shapes were presented at four different sizes to create the following size ratios-1:1, 1:1.5, 1:2, and 1:2.5. The approximate visual angle of the shapes (width $\times$ height) was $2.0^{\circ} \times 2.3^{\circ}$ at Size 1 , $2.9^{\circ} \times 3.5^{\circ}$ at Size $1.5,3.9^{\circ} \times 4.6^{\circ}$ at Size 2 , and $4.9^{\circ} \times 5.8^{\circ}$ at Size 2.5. The shapes were displayed as white outlines on a dark background on an Electrohome ECM 1302 RGB monitor driven by an Apple II+ microcomputer. The center of each shape was approximately $2.4^{\circ}$ to either side of fixation.

Procedure. On each trial two shapes were displayed simultaneously on either side of fixation. The task was to decide whether the two shapes were the same or different as quickly as possible, while ignoring a possible size difference and while keeping errors to a minimum. On every trial, one of the shapes was presented at the smallest size and the other shape at one of four possible sizes $(1,1.5,2$, or 2.5 times the smallest size). The smaller shape was presented to the left of fixation as frequently as to the right, using a random presentation order. ${ }^{1}$

\footnotetext{
' Some readers will have noted that the design used in the present experiment (and in Experiments 2 and 3) confounds the absolute size of the stimuli with the ratio of sizes. However, there have been numerous previous experiments that have examined the issue of whether absolute or relative size is relevant in the experimental design used in the present study (Besner, 1983; Besner \& Coltheart, 1975, 1976; Bundesen \& Larsen, 1975; Bundesen, Larsen, \& Farrell, 1981; Kubovy \& Toth, 1985; Larsen, 1985; Larsen \& Bundesen, 1978; Sekuler \& Nash, 1972). In every case, size ratio rather than absolute size was found to determine mean response time. The range of absolute sizes used in our experiments falls well within that for which absolute size was found not to be important in the above studies. Therefore, the present results are best thought of as resulting from the ratio of sizes between the stimuli rather than from the absolute size of the larger stimulus in each pair.
} 
There was a total of 160 trials for each subject, 80 same and 80 different, divided equally across the four size ratios. ${ }^{2}$ For each size ratio, different trials were created by presenting each shape once at the smallest size and pairing it with another shape, selected at random from the other 19. This other shape was displayed at the appropriate size for that particular size ratio. Same trials were created in the same manner by pairing a shape with itself once at each size ratio.

The trials were ordered at random with the constraint that no more than four consecutive trials required the same response or had the same size ratio. Different random orders were used for each subject.

\section{Results}

Response times. The mean response time for correct responses was computed for each size ratio, each response cate-

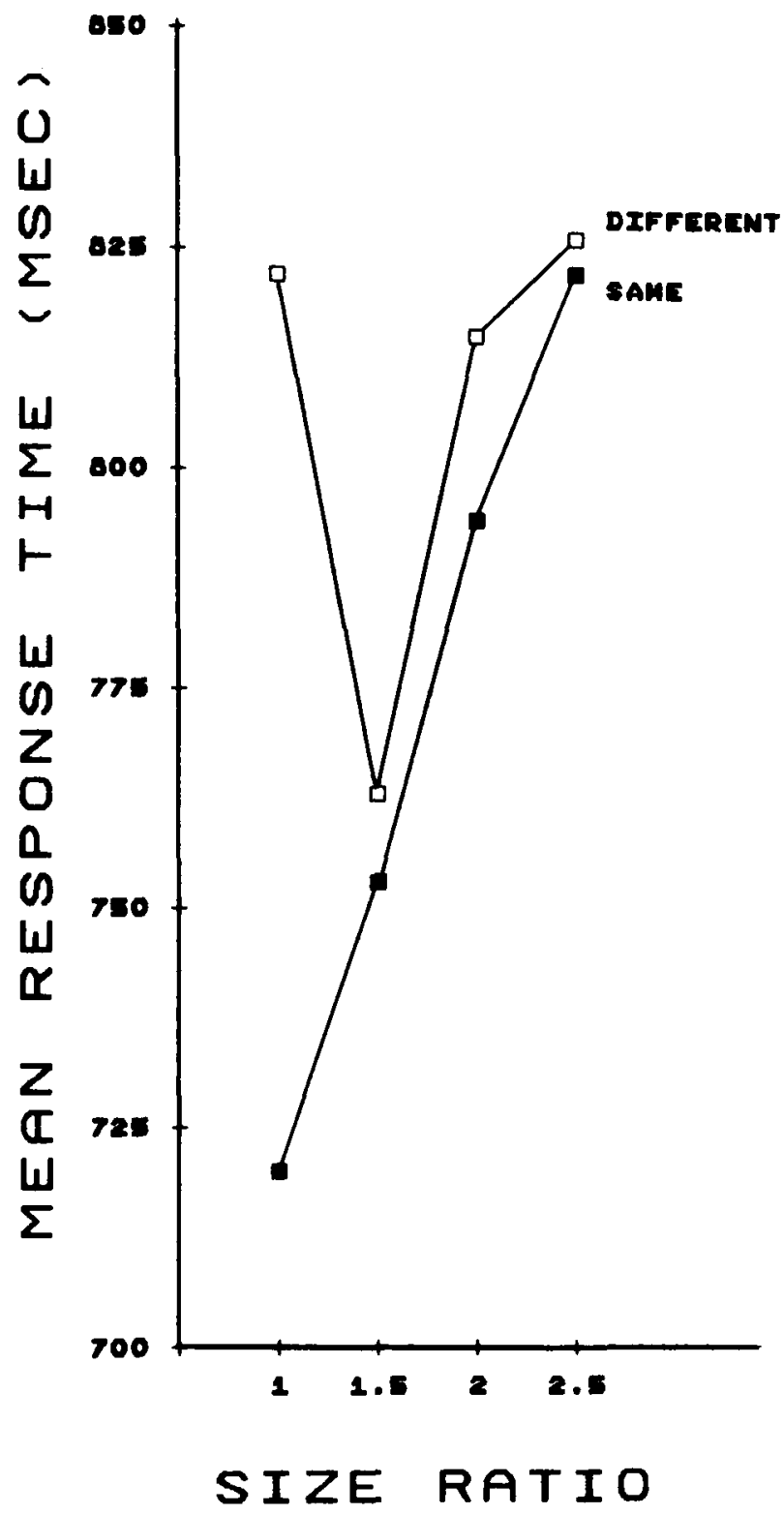

Figure 2. Mean response time, in milliseconds, at each size ratio for same (filled squares) and different (unfilled squares) trials in Experiment 1.
Table 1

Mean Percentage of Error Rate for Each Size Ratio in Same and Different Trials in Experiment 1

\begin{tabular}{lcccc}
\hline & \multicolumn{4}{c}{ Size ratio } \\
\cline { 2 - 5 } Trial type & $1: 1$ & $1: 1.5$ & $1: 2$ & $1: 2.5$ \\
\hline Same & 3.75 & 3.25 & 5.25 & 4.50 \\
Different & 8.50 & 8.00 & 5.25 & 8.75 \\
\hline
\end{tabular}

gory, and each subject; these means are displayed in Figure 2. The means were submitted to a repeated measures analysis of variance in which response type (same/different) and size ratio were considered as factors. There was a large effect of size ratio, $F(3,57)=10.32, p<.0001$, with a strong linear component, $F(1,19)=22.20, p<.0002$. This effect of size ratio, however, was different for the two response categories, $F(3,57)=6.21$, $p<.0015$, which was also reflected in different slopes of the regression lines for the two responses, $F(1,19)=9.19, p<.007$.

As can be seen in Figure 2, the results are additive across same and different trials if one considers just those trials in which the shapes had different sizes, which was confirmed in a separate repeated measures analysis of variance in which only these size ratios were included (i.e., 1.5, 2, and 2.5). In this analysis there was a strong effect of size ratio, $F(2,38)=13.80, p<$ .0001 , which again had a strong linear component, $F(1,19)=$ $28.47, p<.0001$. However, there was no hint of a difference in the pattern of means across response categories in the overall analysis or when considering the slopes of the least squares regression lines $(F<1)$ in both cases.

Errors. Error rates were also computed for each subject, response category, and size ratio. The average error rates are listed in Table 1. The percent error rates were submitted to a repeated measures analysis of variance in which response type (same) different ) and size ratio were considered as factors. There were more errors in different trials (7.6\%) than in same trials $(4.2 \%)$, $F(1,19)=11.89, p<.003$. Error rates were roughly constant across size ratios $(F<1)$, and there was no difference in the pattern of error rates between response categories, $F(3,57)=$

\footnotetext{
${ }^{2}$ Bundesen and Larsen (1975; Larsen, 1985; Larsen \& Bundesen, 1978) do not report results for different pairs when these pairs include distinct shapes. Their rationale for doing so is their claim that size ratio for such pairs is undefined. We find this argument strained, given their explicit belief that the rough size of a shape is determined prior to shape comparison and is used to guide size-scaling operations. Furthermore, it seems relatively easy to create reasonable definitions of size ratiofor example, using notions such as bounded area for closed shapes or length of maximum elongation for open curves-as long as certain "pathological" cases (e.g., very long and narrow shapes) are not included in the stimulus set. In this article the size ratio for different pairs was defined as the size ratio of the matrix within which the shapes were defined-all shapes were stored in the shape table of an Apple II + computer in a $19 \times 19$ pixel array. The outline of every shape touched the outer edge of the array somewhere along each of the sides of the array, which ensured that all the shapes were roughly the same size at a particular scale. Each shape was displayed by scaling the shape in the table before it was displayed on the monitor.
} 
$1.57, p>.20$. As is evident from Table 1 , there is no indication that the pattern of mean response times was due to speed-accuracy trade-offs.

\section{Discussion}

When all size ratios are considered simultaneously, there is a striking difference in the pattern of mean response times for same versus different trials, which replicates one aspect of the results of earlier experiments (Besner, 1983; Besner \& Coltheart, 1975, 1976). As can be seen in Figure 2, however, the difference in the pattern of results appears due to a particularly slow mean response time for different trials when the shapes are shown at the same size. ${ }^{3}$ When shapes are shown at different sizes, the same pattern of means is obtained for same and different trials. That is, mean response time increases at the same rate for both same and different responses.

A preliminary account. In this section we outline a preliminary model designed to account for the pattern of results obtained in the present experiment and in previous studies of the size-ratio effect. Two general findings must be accounted for: First, when different pairs are created by combining different shapes, the results for different size ratios are additive only across same and different trials for larger size ratios. For smaller size ratios, and in particular when the shapes are at the same size, different responses are slower than expected. Second, when different pairs are created by combining rotated versions of the same shape, the results are additive across the entire range of size ratios.

To account for these findings it may be supposed that two factors underlie performance in the usual shape matching paradigm with size-discrepant stimuli. The first factor is the sizescaling operation suggested by Posner and Mitchell (1967), Sekuler and Nash (1972), Corcoran and Besner (1975), Bundesen and Larsen (1975), Larsen and Bundesen (1978), and Larsen (1985). This factor is generally responsible for the increase in response time across size ratios. It operates before the shapes are compared in detail and before the same-different decision is made. The second factor is a form of bias that can slow down the usual course of processing under certain conditions. We argue that in the usual shape-matching experiment using size-discrepant stimuli this bias factor affects different responses when different pairs are composed of different shapes and that the effect is confined to small size ratios.

Our hypothesis is based on the notion that shape comparison processes are sensitive to the context in which the comparisons are carried out. We assume that under usual circumstances the shapes in different pairs composed of rotated versions of the same shape are more similar to each other than are the shapes in different pairs composed by pairing different shapes. This is not to say that different pairs composed of different shapes could not be created in such a way that the shapes in the pairs would be more similar than for rotated versions of the same shape; we say only that this has not been the case to date. In fact, there is evidence to support our assumption: Besner (1983; Experiment 3) found that different pairs composed of rotated shapes were responded to more slowly than different pairs composed of distinct shapes when both types of different pairs were included in the same experiment. In our view, therefore, differ- ent trials in experiments in which different pairs involve distinct shapes occur in a context in which the shapes are more easily discriminated than in experiments in which different pairs involve rotated versions of the same shape.

In the context of easier same-different discriminations, evidence suggesting sameness on a different trial could have a stronger impact on shape-comparison processes than when the context involves relatively difficult discriminations. The impact would be to slow down these different decisions. This is what we think happens on different trials at very small size ratios in the easy discrimination context. The fact that the shapes are shown at the same size increases their similarity in a context in which it is unusual to have evidence for sameness (at least compared with a context in which the same-different discrimination is more difficult). The unusual added sameness evidence slows down the different response and produces the unusually slow response time at small size ratios (see size ratio 1 in Figure 2).

\section{Experiment 2}

The results of Experiment 1 are consistent with the notion that different responses are inhibited when the shapes are shown at the same size. In Experiment 2 we tested the hypothesis proposed in the Discussion of Experiment 1 for the slow different responses to same-sized shapes-namely, that these slow responses are due to a form of bias operating in a particular context. If our analysis is correct, we should be able to reduce or eliminate the inhibition effect on different responses to samesized shapes by an appropriate change in context. In the present experiment we tested this notion by presenting the same pairs of shapes as seen in Experiment 1, with one difference: The trials were blocked by size ratio.

If the slow responses to same-sized different pairs are due to a bias effect engendered by a relative rarity of evidence for sameness, then this inhibition should not be found in a situation in which trials are blocked by size. In the block in which the shapes are shown at the same size, all pairs are accompanied by a match along the dimension of size, including all different pairs. In this context, a match on the size dimension should not be treated as evidence for sameness. On the assumption that two main factors underlie performance in the task-size scaling and bias-and that the blocked procedure minimizes the contribution of the bias factor, we predict equal effects of size discrepancy on same and different responses.

\section{Method}

Subjects. The subjects were 24 undergraduates at the University of Waterloo who participated for pay. All subjects had normal or cor-

\footnotetext{
${ }^{3}$ The careful reader will have noted that the results were somewhat different than expected. Besner (1983; Experiment 2) did not observe additivity across response categories for size discrepancies as small as 1:1.5. In fact, we had hoped to obtain an additive pattern of means across size ratios 1:2 and 1:2.5 and an interactive pattern when all size ratios are considered simultaneously. We can only speculate as to the reasons for the minor difference in results between experiments. Perhaps the actual set of size ratios used in a particular experiment affects the range of size ratio over which subjects will be biased against responding "different."
} 
rected-to-normal vision, and none of the subjects participated in Experiment I or 3.

Stimuli. The stimuli, presentation apparatus, and viewing conditions were the same as in Experiment 1.

Procedure. The procedure was identical to that used in Experiment 1 except for differences engendered by the presentation of trials blocked according to the size difference between the shapes. There were four blocks of trials, one for each size ratio (1:1, 1:1.5, 1:2, and 1:2.5). Each block consisted of 40 trials-20 same and 20 different-created as in Experiment 1, which were preceded by 10 practice trials. A different order of the four blocks was used for each subject, and across subjects every possible block order was used once. The trial sequence within each block was determined at random for each subject.

\section{Results}

Response times. The mean response time for correct responses was computed for each response type (same/different), each block (size ratio), and each subject. The mean of these means is shown in Figure 3 for each size ratio and each response category. The means were submitted to a repeated measures analysis of variance in which size ratio and response type (same/different) were considered as factors.

The only significant factor in the analysis reflected the effects of size ratio; response times increased as size ratio increased, $F(3,69)=6.08, p<.001$. The increase across size ratios had a strong linear component, $F(1,23)=12.60, p<.002$. Unlike the results for Experiment 1, there was no difference in the effect of size ratio between same and different responses in the overall analysis, $F(3,69)=1.20, p>.31$, or in the analysis of linear trends $(F<1)$. The difference in mean response time between same and different responses was not significant, $F(1,23)=$ $2.43, p>.13$.

We also compared the results from this experiment with those in Experiment 1, using a mixed design analysis of variance in which experiments was a between-subjects factor and size ratio was a within-subjects factor. When considering only different responses, the pattern of means was different across the two experiments, $F(3,126)=2.43, p<.007$, which indicates a significant change in pattern, depending on the experimental context. In contrast, there was no comparable difference for same responses across experiments $(F<1)$.

Errors. The percentage of errors for different response types (same/different) and blocks (size ratios) for each subject were analyzed in a repeated measures analysis of variance in which response type and size ratio were the factors. The mean error rate for each response and size ratio can be seen in Table 2.

There were more errors on different trials than on same trials, $F(1,23)=8.90, p<.007$. There were no other significant effects $(p>.35)$ in all cases. A visual inspection of the error rates in Table 2 reveals that the general increase in response time across size ratios is not due to speed-accuracy trade-offs. However, note that for same responses the 1:1.5 size ratio condition yielded the highest error rate, which may indicate some tradeoff between speed and accuracy, given that this condition was also associated with the fastest mean response time. For this reason, we will not consider further the slightly anomalous pattern of mean response times for same responses.

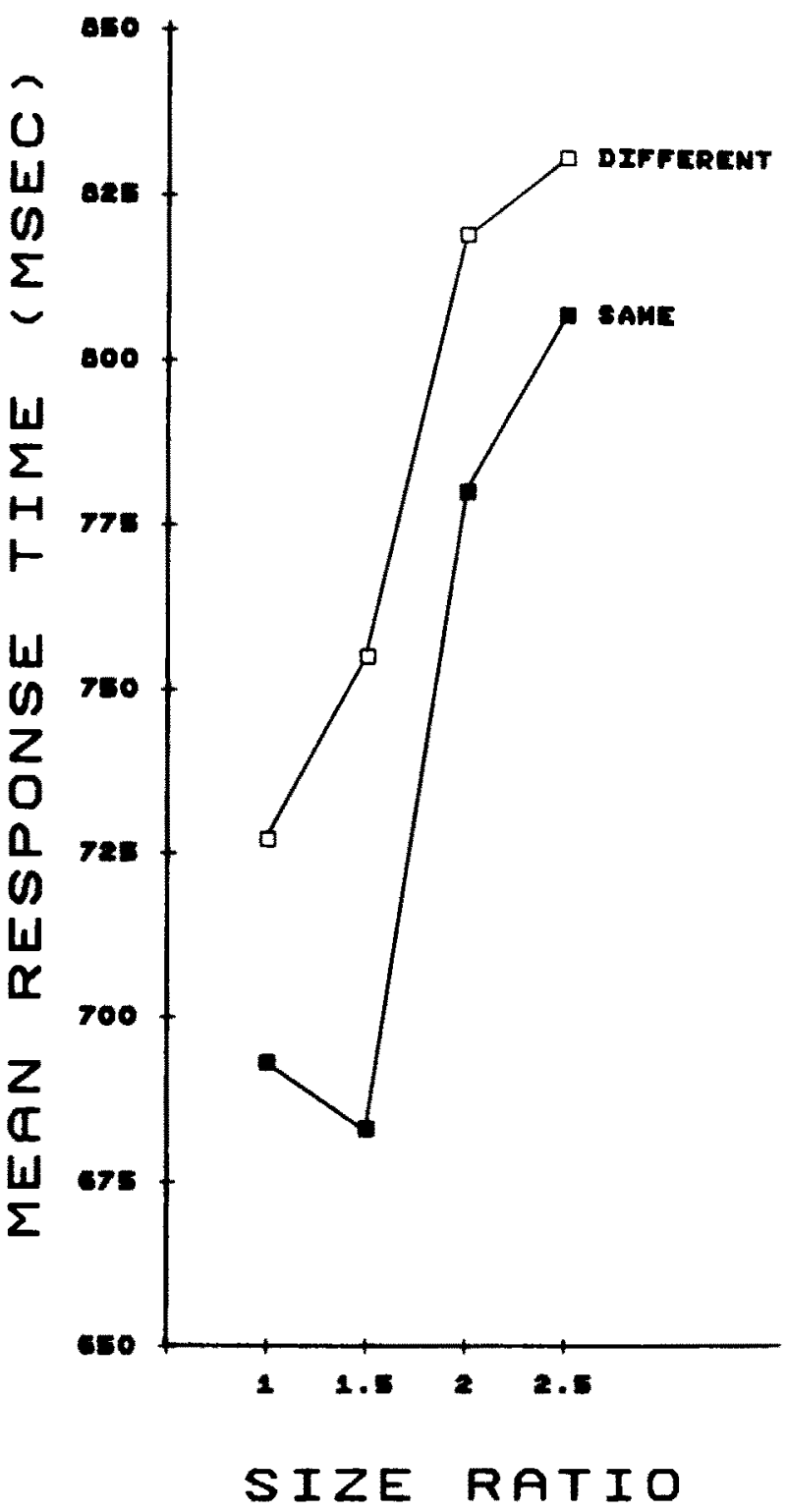

Figure 3. Mean response time, in milliseconds, at each size ratio for same (filled squares) and different (unfilled squares) trials in Experiment 2 .

\section{Discussion}

The most important results were clear-cut: Response times increased monotonically across size ratios for different responses, as expected if the nonmonotonic effects of size in Experiment 1 were due to a form of bias. These are the most important results because they support the notion that different responses in the usual size-disparity paradigm can be strongly influenced by a bias mechanism. Same responses, on the other hand, continued to be strongly influenced by size ratio, and the size ratio effect was not statistically different across the two experiments.

The fact that both same and different response times increased sharply with increasing size ratio is consistent with the 
Table 2

Mean Percentage of Error Rate for Each Size Ratio in Same and Different Trials in Experiment 2

\begin{tabular}{lllll}
\hline & \multicolumn{4}{c}{ Size ratio } \\
\cline { 2 - 5 } Trial type & $1: 1$ & $1: 1.5$ & $1: 2$ & $1: 2.5$ \\
\hline Same & 4.17 & 4.79 & 3.96 & 4.17 \\
Different & 6.88 & 6.67 & 9.79 & 7.71 \\
\hline
\end{tabular}

supposed operation of an underlying size-scaling mechanism. These results do not support the view that size-ratio effects on same trials reflect nothing more than response bias (Besner \& Coltheart, 1976). On this hypothesis, size-ratio effects should have been sharply reduced in the present experiment, given that the trials were blocked by size ratio. It is not clear why subjects would not adjust their criterion for responding same so as to take into account the fact that the pairs in a particular block would involve a given size difference. Evidently, when every pair in a block of trials involves the same size difference and when this difference is associated equally often with a same response as with a different response, the size discrepancy does not count as evidence that the shapes are different.

\section{Experiment 3}

The results so far can be explained by the operation of two underlying mechanisms: a size-sensitive mechanism responsible for the increase in response time as size ratio is increased (such as size scaling) and a bias mechanism modulating the response time for different responses when shapes have the same or nearly the same size. Experiment 3 was designed to provide additional evidence for the notion that the shape of the response time function across size discrepancy reflects the operation of these two mechanisms. Furthermore, we wished to demonstrate the effect of bias on same responses as well as on different responses.

To demonstrate the operation of bias on same decisions, we included another manipulation in the experimental design. The stimuli were modified by adding a pattern of light and dark regions within the bounding contour of each shape. Not only could the two shapes be the same or different, but the pattern of light and dark within each contour could also be the same or different. These two variables were varied orthogonally. However, subjects were instructed to base their decisions only on whether the bounding contour of the shapes was the same or different, while ignoring the light/dark shading pattern and while also ignoring size.

The rationale for the inclusion of the light/dark shading inside the shapes hinges on the hypothesis that subjects will not always be able to ignore the internal shading pattern when making decisions about the shape of the bounding contour. In general, differing shading patterns should bias subjects to respond "different," and identical shading patterns should bias subjects to respond "same." On trials in which the bounding shapes are the same, different shading patterns should slow the execution of the required same response. Given that the similarity of the

shading pattern should be most readily apparent when the shapes are shown at the same size, this bias effect should be most pronounced for pairs of shapes shown at the same size. Conversely, when the bounding contours have different shapes, identical internal shading patterns should slow the required different response, and this effect should be most apparent when the shapes are shown at the same size.

The second underlying process, which is sensitive to size ratio, should continue to operate when the shapes are shown in different sizes and continue to produce response times that are increasingly longer as the size ratio is increased for both same and different trials.

\section{Method}

Subjects. The subjects were 20 undergraduates at the University of Waterloo who were paid for their participation. All subjects had normal or corrected-to-normal vision. No subject in this experiment participated in Experiment 1 or 2.

Stimuli. The shapes used in Experiments 1 and 2 were employed in this experiment as the bounding contours of the shapes. For each basic shape, 15-20 rectangular regions within the bounding contour of the shape were designated as allowable regions for filling in. On each trial, a random subset of these regions was shown at the same intensity as the bounding contour (white), and the remaining regions were displayed at the lower background intensity (black). Example stimulus displays are shown in Figure 4.

Procedure. On each trial two shapes were presented simultaneously to either side of fixation. The task was to decide as quickly as possible

SAnE SHAPE

SAMC OMADzNo

SAME CHAPT DIFFERET sMadzue GAME omabimo DxFrentut OMAPE
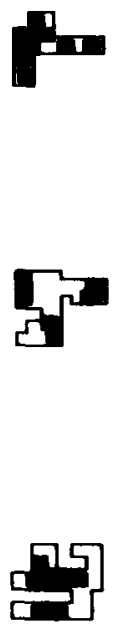

DIPrCERT OMAPL

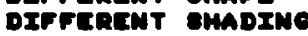
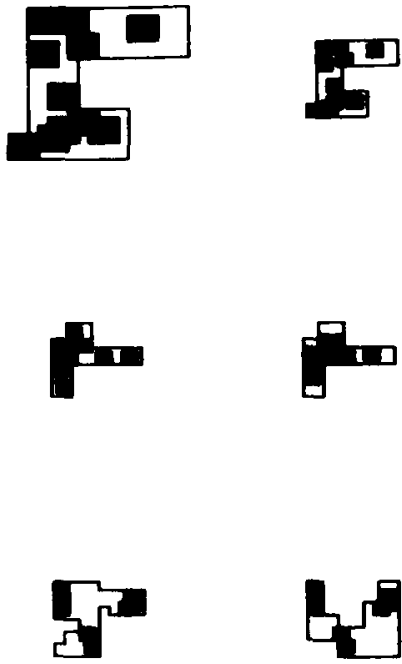

Figure 4. Example stimuli used in Experiment 3. (Note that the displays were shown to subjects as white lines and blocks on a black background.) 
whether the two shapes were the same or different while keeping errors to a minimum and while ignoring a possible size difference and a possible difference in the internal light/dark shading pattern.

There were 16 types of trials resulting from the factorial combination of three factors: shape of the bounding contour (same or different), shading pattern inside the shape contour (same or different), and size ratio $(1: 1,1: 1.5,1: 2$, or 1:2.5). On trials in which the shapes were the same, same-shading patterns were produced by selecting a random subset of internal regions to be displayed at high intensity, and "painting in" the same regions in both shapes. The entire pattern for one of the shapes was then scaled to the appropriate size, if necessary. For the same-shapes/different-shadings condition, the random selection of shading regions was carried out independently for each shape. When the two bounding shapes were different, the following procedure was employed. First, the two shapes were overlapped (all shapes were defined relative to a $19 \times 19$ pixel array), and the set of allowable shading regions common to both shapes was extracted. In same-shading trials, a random selection from the set of common regions was selected for highlighting, and this pattern was used in both shapes. In different-shading trials, different random selections from the set of common regions were used in both shapes.

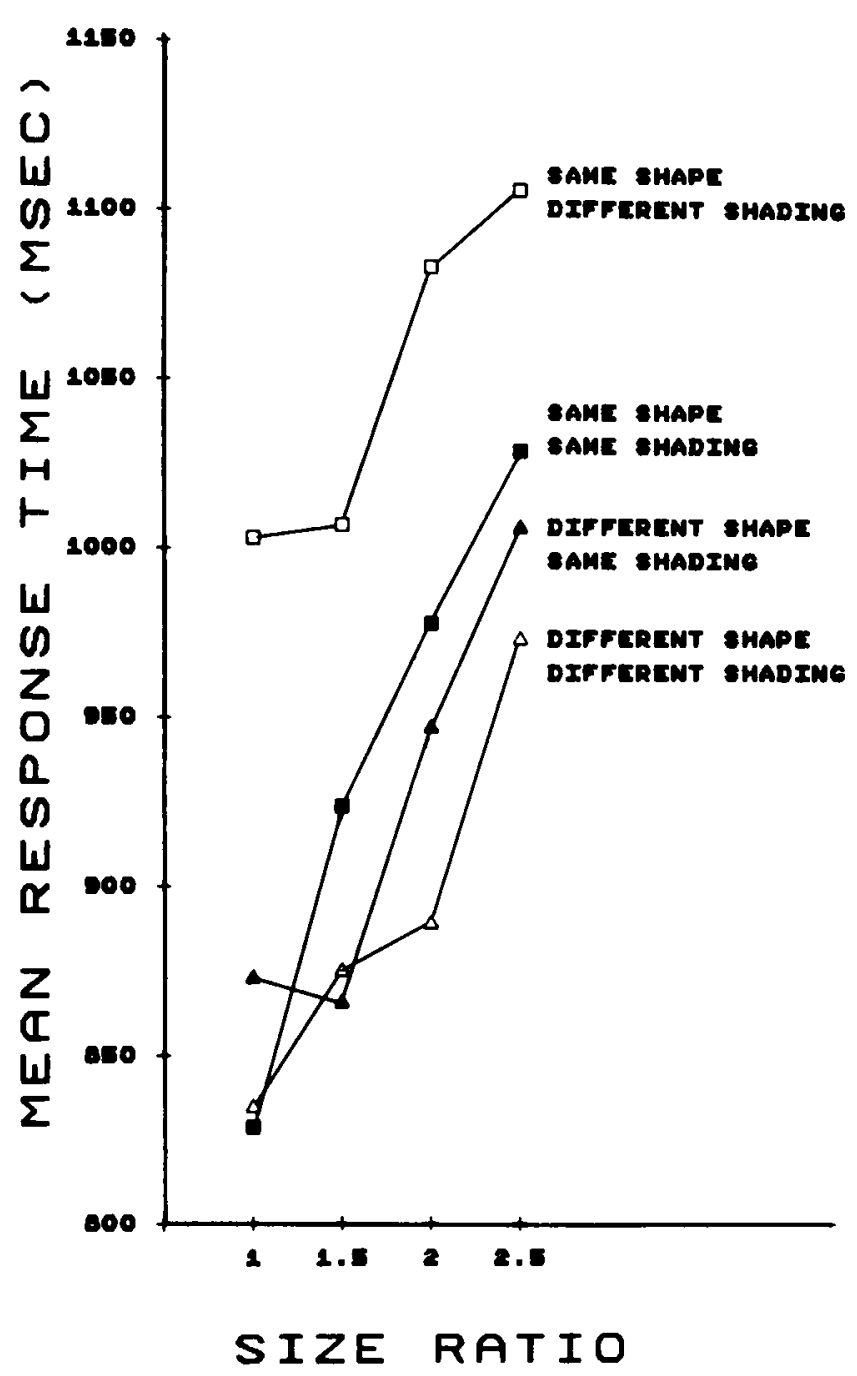

Figure S. Mean response time, in milliseconds, at each size ratio in each condition of Experiment 3.
Table 3

Mean Response Time ( $R T$, in Milliseconds) and Percentage of the Error Rate for Trials With Same or Different Shapes and Same or Different Shading Patterns in Experiment 3

\begin{tabular}{lccccc}
\hline & \multicolumn{2}{c}{ Same shape } & & \multicolumn{2}{c}{ Different shape } \\
\cline { 2 - 3 } \multicolumn{1}{c}{ Condition } & RT & Error & & RT & Error \\
\hline Same shading & 940 & 5.1 & & 923 & 7.4 \\
Different shading & 1,050 & 7.9 & & 893 & 6.4 \\
\hline
\end{tabular}

The size ratios and presentation conditions were identical to those used in Experiment 1 except for minor differences due to the addition of internal shading as a factor. There were 320 trials in all- 20 trials in each of the 16 conditions-and a brief rest period was included after the first 160 trials. As in Experiment 1, different random orders of trials were used for each subject, and trials with different size ratios were intermixed at random.

\section{Results}

Response times. The response times for correct responses were averaged for each subject, each size ratio, each shading condition (same/different), and each response (same/different). These means were submitted to a repeated measures analysis of variance in which shading, response, and size ratio were considered as factors. Figure 5 displays the mean of the means for each size ratio, response, and shading condition. As expected, the overall mean response time increased sharply across size ratios, $F(3,57)=31.29, p<.0001$, with a strong linear component, $F(1,19)=37.85, p<.0001$.

The results provide clear evidence for the operation of bias. First, the response factor (same/different bounding shape) and the shading factor (same/different shading pattern) had significant overall effects when taken singly, $F(1,19)=5.16, p<.04$ for shape, and $F(1,19)=14.57, p<.002$ for shading; these two variables also entered into an interaction with each other, $F(1$, $19)=56.63, p<.0001$. The relevant means are listed in Table 3. When the shapes were the same, responses were faster when the shading patterns were also the same than when they were different. In contrast, when the shapes were different, responses were faster when the shading patterns were different than when they were the same. This pattern of results provides clear-cut evidence for the notion of bias. When all the available evidence from the bounding shape and the shading pattern was consistent, being either both the same or both different, response times were relatively fast. When the evidence from the shapes and shading patterns conflicted, responses times were relatively slow. These results suggest that although subjects were instructed to respond only on the basis of the shapes of the bounding contours, they did not ignore completely the information contained in the internal sharing patterns (see also Hawkins \& Shigley, 1972).

A second source of evidence for the effect of bias can be found by examining the results across size ratios, particularly for trials in which the shapes (and thus the shading patterns) had the same size. First consider the results for trials in which the shading patterns were the same (the filled symbols in Figure 5). 
These results are similar in many respects to those found in Experiment 1 . Response times increase regularly across size ratios for same responses (there is even a suggestion that same responses at size ratio 1 were faster than expected from the linear extrapolation of the results for the larger size ratios), whereas we again find an elevated response time in different trials when the shapes had the same size (although not nearly as much as in Experiment 1). This is exactly what we would expect if the presence of identical shading patterns facilitated same decisions and inhibited different responses when the patterns had the same size. Otherwise, the response times appear additive across same and different trials for different size ratios (from 1.5 to 2.5 ).

Now consider the results for trials in which the shading patterns were different (unfilled symbols in Figure 5). In this case, response times increase monotonically across size ratio for different responses and show a similar pattern for same responses, but with a somewhat elevated mean for same-sized shapes. This pattern of means would be expected if the difference in shading pattern facilitated different decisions and inhibited same decisions, especially when the patterns were shown at the same size.

Another way to look at these results is to consider the effect of shading on same or different responses. In general, when the shading patterns across the two shapes were consistent with the response, faster mean times were observed; in contrast, when the shading patterns were inconsistent with the response, response times were slower. Furthermore, this pattern was especially marked when the shapes were shown at the same size.

The pattern of results described above should be reflected in the overall analysis of variance by the three-way interaction among size ratio, shape, and shading, which was in fact significant, $F(3,57)=3.18, p<.031$. Two additional analyses of variance were carried out to confirm more precisely the above observations. In the first analysis, we considered only the first two size ratios (1 and 1.5). Most importantly, this analysis revealed a three-way interaction among size ratio, shape, and shading, $F(1,19)=6.86, p<.02$. This interaction reflects the increased strength of the bias factor at size ratio 1 compared with the effect at ratio 1.5. In the second analysis, we considered only the results for trials in which the two shapes had different sizes (size ratios $1.5,2$, and 2.5 ). This analysis confirmed the roughly additive appearance of the results for these size ratios: The threeway interaction was not significant, $F(2,38)=2.09, p>.13$, which was also true when the slopes of the regression lines across size ratios were compared $(F<1)$.

There were no other significant effects in the overall analysis of variance; both the Shape $\times$ Size interaction and the Shading $X$ Size interaction were not statistically significant $(p>.16$ in both cases).

As can be seen by comparing the results in Figures 2 and 5 , the size ratio effect appears somewhat larger in Experiment 3 than in Experiment 1. Response times also seem generally longer in Experiment 3 than in Experiment 1. A final analysis was carried out to compare the results from these two experiments in which size ratios were intermixed at random. In this analysis we included only the results for trials in which the shapes differed in size (size ratios 1.5, 2, and 2.5) because these results appear to reflect more clearly the influence of possible
Table 4

Mean Percentage of Error Rate for Each Response (Same or Different Bounding Shapes), Shading Condition. and Size Ratio in Experiment 3

\begin{tabular}{lrlll}
\hline & \multicolumn{4}{c}{ Size ratio } \\
\cline { 2 - 5 } \multicolumn{1}{c}{ Condition } & $1: 1$ & $1: 1.5$ & $1: 2$ & $1: 2.5$ \\
\hline Same shape & & & & \\
$\quad$ Same shading & 4.75 & 4.25 & 6.00 & 5.25 \\
$\quad$ Different shading & 10.25 & 6.75 & 7.50 & 7.00 \\
Different shape & & & & \\
$\quad$ Same shading & 5.75 & 7.25 & 7.75 & 8.75 \\
$\quad$ Different shading & 5.00 & 5.75 & 7.75 & 7.25 \\
\hline
\end{tabular}

scaling processes with less influence from bias mechanisms. In both experiments we averaged results from all conditions for each size discrepancy. These means were submitted to a mixeddesign analysis of variance in which experiment was a betweensubjects factor and size was a within-subjects factor. As suggested by a visual comparison of the size-discrepancy effects displayed in Figures 2 and 5 , the overall effect of size discrepancy was larger in Experiment 3 than in Experiment 1, F(2, 76) $=3.14, p<.05$, as was the average slope of the least-squares lines, $F(1,38)=4.61, p<.04$. The overall mean for these size ratios also was larger in Experiment $3(974 \mathrm{~ms})$ than in Experiment 1 (796 ms), $F(1,38)=5.62, p<.023$.

Errors. The average percentage of errors for each response, each shading condition, and each size ratio is presented in Table 4. These error rates were also submitted to a repeated measures analysis of variance in which response, shading, and size ratios were the factors.

Only two effects in the analysis of variance approached significance. The first was the Shape $\times$ Shading interaction, $F(1$, $19)=3.47, p<.08$. As we would expect from the analysis of response times, trials in which both the shape and the shading were the same and triais in which both were different tended to yield smaller error rates. Thus, the pattern of mean response times for this effect was not due to a speed-accuracy trade-off. The second nearly significant effect was due to different patterns of errors across size ratios for different responses, $F(3,57)=$ $2.60, p<.062$. As can be seen in Table 4, error rates tended to increase with size ratio when the bounding shapes were different; in contrast, they tended to decrease when the shapes were the same. Given that this effect was only marginally significant and that it was not found in Experiments 1 or 2, we will not consider it further. In any case, the pattern of error rates does not suggest that the response time results were due to speedaccuracy trade-offs.

\section{Discussion}

The results were as expected if two factors underlie performance when shapes that differ in size are matched perceptually. One factor appears to be a continuous size scaling process. In this view, a representation of one of the two shapes is transformed in a gradual and analog fashion so as to compensate for the difference in size between the two shapes, at which point 
they are compared. This scaling operation is thought to take more time when the ratio of sizes between the two shapes is larger because the process is analog. This process is reflected in the results by the general increase in response times as the size discrepancy between shapes is increased. The other factor is some form of bias. The presence of mismatching internal shading information, whether relevant or irrelevant to the correct response, tends to slow down the output of same responses and to speed up the output of different responses. Similarly, the presence of matching internal shading information tends to speed up same responses and to slow down different responses.

The irrelevant shading patterns had substantial effects at all levels of size discrepancy. In fact, the results were additive across the various conditions from size ratios 1.5 to 2.5 , which were the trials involving different-sized stimuli. These results suggest that portions or all of the internal shading patterns were scaled and compared in the perceptual matching process. As is evident in Figure 5, however (for example, consider the results for same-shape/different-shading trials, unfilled squares), the effects of the irrelevant internal shading had marked effects at every size ratio (compare with results for same-shape/sameshading trials, filled squares). If size-scaled images of the shapes were compared, as suggested by Bundesen and Larsen (1975; Larsen, 1985; Larsen \& Bundesen, 1978), then these images included some or all of the information present in the original stimuli, including the shading pattern-subjects did not scale and compare only "skeletal" images (see Kosslyn, 1980) of the original stimuli. The fact that the magnitude of the size discrepancy effect was larger in Experiment 3 than in Experiment 1 is consistent with the notion that more complex shapes are scaled more slowly than simpler shapes. These results lend further support to the notion that the scaling processes used in Experiment 3 operated on representations that included some or all of the internal shading pattern, given that this was the only difference between the shapes in Experiment 1 and Experiment 3. In addition, these results could point to important differences between processes that compensate for size differences and those that compensate for orientation differences in shape matching experiments, given that complexity effects on the rate of compensation have been elusive in the mental rotation literature (see for example, Shepard \& Cooper, 1982; but see also Bethell-Fox \& Shepard, 1985).

The bias effects have their strongest influence when shapes are shown at the same size (see Figure 5). It is possible that shapes shown at the same size can be compared more efficiently (perhaps via "direct" comparison of visual codes; Simion, Bagnara, Roncato, \& Umiltà, 1982) than when they are compared via mental images, which would enhance the effects of matching and mismatching irrelevant visual information. Another possibility is that there are two forms of bias: one form sensitive to the size ratio of the shapes and to the experimental context, and another form sensitive to other types of matching and mismatching irrelevant attributes. It remains to be determined whether the bias effects observed here have one general underlying source or whether there are two distinct types of bias.

\section{General Discussion}

Several experiments in the literature suggest that the ratio of size difference between shapes does not influence the speed of different judgments when different trials are created by pairing different shapes (Besner, 1983; Besner \& Coltheart, 1975, 1976). These results have been used to argue that mental size scaling either is not a necessary operation prior to the mental comparison of visual shapes or that different processes underlie same and different responses. However, our new results suggest that these differences across response categories are not always good evidence against the operation of a size scaling mechanism. Rather, the nonmonotonicity in the effect of size ratio (especially for different trials) may reflect a bias effect superimposed on the usual linear effects of size ratio.

For expository purposes, we have supposed throughout the article that the size-ratio effect reflects the operation of a continuous size scaling operation, as suggested by a number of researchers (e.g., Bundesen \& Larsen, 1975; Larsen \& Bundesen, 1978; Posner \& Mitchell, 1967; Sekuler \& Nash, 1972). Although the linear effects of size ratio on response times are consistent with the operation of a continuous and analog transformation process, they do not exclude other possible interpretations. Further research will be needed to demonstrate that the size-scaling interpretation of the size-ratio effect is to be preferred over other possible accounts that would also yield linear effects of size ratio. Regardless of the mechanism giving rise to the linear size-ratio effect, our results suggest that all the available evidence is consistent with the view that this effect occurs prior to shape matching operations.

Even if the size-ratio effect is due to a continuous scaling operation, it is clear that this operation is not necessary in all cases. In fact, the size-ratio effect disappears when the shapes are very familiar to the subjects due to repeated presentation in a particular experimental situation (Kubovy \& Podgorny, 1981; Kubovy \& Toth, 1985; Larsen, 1985).

Our general conclusion is that a closer examination of the role of bias may be particularly important for a more detailed understanding of the size-ratio effect, which should in turn enhance our understanding of perceptual comparisons in general.

\footnotetext{
A A potential test of the notion that there are different bias effects might be to repeat Experiment 3, but with the shading variable manipulated between blocks. If the shading effects persisted in this experiment, this bias effect would be different from the bias effect that disappeared by blocking trials by size ratio (i.e., from Experiment 1 to Experiment 2).
}

\section{References}

Besner, D. (1978). Pattern recognition: Are size and orientation additive factors? Perception \& Psychophysics, 23, 93.

Besner, D. (1983). Visual pattern recognition: Size preprocessing reexamined. Quarterly Journal of Experimental Psychology, 35A, 209216.

Besner, D., \& Coltheart, M. (1975). Same-different judgments with words and nonwords: The differential effects of relative size. Memory \& Cognition, 3, 673-677.

Besner, D., \& Coltheart, M. (1976). Mental size scaling examined. Memory \& Cognition, 4, 525-531.

Bethell-Fox, C. E., Shepard, R. N. (1985, November). Mental rotation: Effects of stimulus complexity, familiarity, and individual differences. Paper presented at The Twenty-Sixth Annual Meeting of The Psychonomic Society, Boston.

Bundesen, C., \& Larsen, A. (1975). Visual transformation of size. Jour- 
nal of Experimental Psychology: Human Perception and Performance, 1, 214-220.

Bundesen, C., Larsen, A., \& Farrell, J. E. (1981). Mental transformation of size and orientation. In A. Baddeley \& J. Long (Eds.), Attention \& performance IX. (pp. 279-294). Hillsdale, NJ: Erlbaum.

Corcoran, D. W. J., \& Besner, D. (1975). Application of the Posner technique to the study of size and brightness irrelevancies in letter pairs. In P. M. A. Rabbitt \& S. Dornic (Eds.), Attention and performance V. (pp. 613-629). London: Academic Press.

Hawkins, H. L., \& Shigley, R. H. (1972). Irrelevant information and processing mode in speeded discrimination. Journal of Experimental Psychology, 96, 389-395.

Howard, J. H., \& Kerst, S. M. (1978). Directional effects of size changes on the comparison of visual shapes. Journal of Experimental Psychology, 91, 491-499.

Kosslyn, S. M. (1980). Image and mind. Cambridge, MA: Harvard University Press.

Kubovy, M., \& Podgorny, P. (1981). Does pattern matching require the normalization of size and orientation? Perception \& Psychophysics, 30, 24-28.

Kubovy, M., \& Toth, N. (1985, November). Parallel vs. serial mental transformations. Paper presented at The Twenty-Sixth Annual Meeting of The Psychonomic Society, Boston.

Larsen, A. (1985). Pattern matching: Effects of size ratio, angular difference in orientation, and familiarity. Perception \& Psychophysics, 38, 63-68.

Larsen, A., \& Bundesen, C. (1978). Size scaling in visual pattern recognition. Journal of Experimental Psychology: Human Perception and Performance, 4, 1-20.

Posner, M. I., \& Mitchell, R. F. (1967). Chronometric analysis of classification. Psychological Review, 74, 392-409.

Sekuler, R., \& Nash, D. (1972). Speed of size scaling in human vision. Psychonomic Science, 27, 93-94.

Shepard, R. N., \& Cooper, L. A. (1982). Mental images and their transformations. Cambridge, MA: The MIT Press.

Simion, F., Bagnara, S., Roncato, S., \& Umiltà, C. (1982). Transformation processes upon the visual code. Perception \& Psychophysics, 31 . 13-25.

Received October 16, 1986

Revision received February 5, 1987

Accepted February 6, 1987 